\title{
Evolutionary Competition Between Prediction Rules and the Emergence of Business Cycles within Metzler's Inventory Model
}

\author{
Michael Wegener and Frank Westerhoff \\ University of Bamberg, Department of Economics, Feldkirchenstrasse 21, D-96045 \\ Bamberg, Germany
}

\begin{abstract}
We revise Metzler's well-known inventory model by developing a framework in which producers can either apply a simple but cheap extrapolative or a more sophisticated but expensive regressive forecasting rule to predict future sales. Producers are boundedly rational in the sense that they tend to select forecasting rules with a high evolutionary fitness. Using a mixture of analytical and numerical tools, we attempt to describe the characteristics of our model's dynamical system. Our results suggest that the evolutionary competition between heterogeneous predictors may lead to irregular fluctuations in economic activity.
\end{abstract}

Keywords: business cycles, inventory adjustment, heterogeneous expectations, learning behaviour, nonlinear dynamics

JEL codes: D84, E12, E32

\section{Introduction}

It has long been known that expected future sales and inventory stock production may play an important role in the motion of national income and the business cycle 1 One of the first models that attempts to capture the interplay between expected future sales, inventory stock production and the business cycle is Metzler's (1941) well-known business cycle model. In this paper, Metzler presents the dynamics that can be derived from a simple linear model in which all producers hold the same heuristically derived expectation on future sales. Overall, his main contribution is to show that in such a framework, inventory adjustments, triggered by erroneous sales expectations, may lead to dampened business cycle fluctuations. It is worth noting that Metzler's linear approach is also able to generate business cycles with constant amplitude. However, this feature merely holds for quite specific parameter combinations.

\footnotetext{
${ }^{1}$ Akerlof and Shiller (2009) convincingly argue that people's expectations are subject to animal spirits and may thus have a destabilising impact on macroeconomic outcomes. Moreover, the relevance of inventory adjustments on the evolution of the business cycle is, amongst others, demonstrated in Blinder and Maccini (1991) or Ramey and West (1997).
}

(C) 2010 Kluwer Academic Publishers. Printed in the Netherlands. 
In this paper, we want to broaden this view by extending Metzler's original model nonlinearly 2 For this purpose, we introduce heterogeneous expectation formation into Metzler's framework. For the sake of simplicity, we will thereby focus on two different types of predictors, i.e. producers either apply a simple but cheap extrapolative or a more sophisticated but also more expensive regressive forecasting rule in order to predict expected future sales. Moreover, producers exhibit a boundedly rational learning behaviour in the sense that they tend to select predictors that produced low squared forecasting errors in the past.

As it turns out, the motion of the business cycle is defined by a higherorder nonlinear difference equation, which has a unique fixed point. Furthermore, we use a mixture of analytical and numerical tools to describe the characteristics of this dynamical system. One central finding is that our model is able to produce complex endogenous dynamics for a broad range of parameter combinations. Such fluctuations are particularly likely if agents react sensitively to differences in the rules' performance. In such a situation, we then observe alternating periods in which the majority of firms either rely on destabilising extrapolative or stabilising regressive expectations. Another interesting finding is that we observe coexisting attractors which emerge due to a so-called Chenciner or crater bifurcation. Depending on initial conditions (or exogenous shocks), national income may then either be driven towards its long-run equilibrium or will display endogenous oscillations.

In a broader context, our paper is related to a number of recent contributions which show that the expectation formation of boundedly rational heterogeneous agents, relying on heuristic forecasting rules, is an important driver of business cycle dynamics. There are several proposals on how to model the agent's predictor selection. In Westerhoff and Hohnisch (2008), agents may change their strategy due to (random) social interactions with other agents, and thus display a kind of herding behaviour. In Lines and Westerhoff (2006) or Wegener et al. (2009), in turn, agents switch between extrapolative and regressive expectations in dependence on how far the economy deviates from its long-run equilibrium, i.e. regressive expectations gain in prominence as booms and slumps continue to grow, which makes an eventual mean reversion more likely. More closely related to our current paper are models in which the selection of forecasting strategies depends on their past performance. For papers in this direction, which employ quite different types of macroeconomic models, see Branch and McGough $(2009,2010)$,

\footnotetext{
${ }^{2}$ For other (nonlinear) extensions of Metzler's framework, see, e.g. Franke and Lux (1993), Franke (1996) or Matsumoto (1998).
} 
Anufriev et al. (2008), DeGrauwe (2008) or Lines and Westerhoff (2010). Some of these models also explicitly incorporate learning behaviour 3 In Berardi (2007), for example, agents adjust the parameters of their forecasting rules via recursive least squares techniques, while Tuinstra and Wagener (2007) construct an interesting framework in which agents switch between two competing learning strategies. Moreover, Marcet and Nicolini (2003) have developed a model of "quasi-rational learning", which is able to replicate certain stylised facts observed during periods of hyperinflation. Overall, these models have at least some ability to explain business cycle dynamics, and may thus also be relevant from a policy perspective. Nevertheless, our paper differs from this strand of research in the sense that we focus on sales expectations of heterogeneous firms. We consider such a focus to be a quite promising and natural starting point for exploring the relevance and consequences of heterogeneous expectations on business cycle dynamics.

This paper is organised as follows. Our model is presented in section 2. In section [3] we begin with some comments on the stability of our dynamical system. Section 4 in turn, contains a specific numerical illustration of how our model functions, while section 5 demonstrates the dynamical behaviour of our model for a broader range of parameter combinations. The last section concludes the paper.

\section{The model}

In Metzler's (1941) business cycle model, national income, $Y$, is determined by the three components: investments, $I$, inventory adjustments, $S$, and expected consumer demand, $U$. Therefore, we have

$$
Y_{t}=I_{t}+S_{t}+U_{t}
$$

To simplify matters, firms' investments are regarded to be fixed, i.e.

$$
I_{t}=\bar{I} .
$$

Moreover, their inventory adjustments, $S$, at time $t$ are given by

$$
S_{t}=\widehat{Q}_{t}-Q_{t-1},
$$

where $\widehat{Q}_{t}$ denotes the desired inventory level producers wish to maintain, and the realised inventory at the close of the previous period is denoted by $Q_{t-1}$. Note that adjustments of the inventory stock can be

\footnotetext{
${ }^{3}$ See Evans and Honkapojah (2001) for a general survey on learning in macroeconomics.
} 
both negative and positive, corresponding to the depletion or accumulation of inventory.

The desired inventory level is simply proportional to the expected sales of consumption goods, i.e.

$$
\widehat{Q}_{t}=k U_{t}
$$

where parameter $k>0$ may be regarded as an inventory accelerator.

Producers may not correctly predict consumer demand and thus do not realise their desired inventory level. Hence, the effectively realised inventory level, $Q$, is determined by the desired inventory level adjusted by unexpected inventory changes. Since these unexpected inventory changes are the differences between realised and expected sales, the effectively realised inventory level at the close of the previous period is determined by

$$
Q_{t-1}=\widehat{Q}_{t-1}-\left(C_{t-1}-U_{t-1}\right) .
$$

Realised sales are given by agents' consumption expenditures, and are assumed to be proportional to national income, i.e.

$$
C_{t}=b Y_{t}
$$

where parameter $b$ denotes the marginal propensity to consume, and is obviously restricted to $0<b<1$.

The framework presented so far followed Metzler's (1941) original model, in which expected consumer demand is homogeneously modelled as the expectation of a single group of producers 4 Now we would like to broaden this homogeneous point of view by introducing two different types of competing predictors that firms may use to determine expected future sales. Note that there is significant empirical evidence supporting the view that agents are boundedly rational and display rulegoverned behaviour 5 Moreover, laboratory experiments by Hommes et al. (2005b) and Heemeijer et al. (2009) indicate that agents tend to use simple linear forecasting rules to form predictions. In particular, agents seem to use both extrapolative and regressive expectation formation rules. There is also evidence that agents change their forecasting strategies over time. Inspecting survey data on inflation expectation, Branch (2004) concludes that agents do not blindly follow a certain rule but that they select between a limited set of different predictors. Interestingly, the proportion of agents using a certain predictor thereby varies inversely with the predictor's mean squared forecasting error, i.e.

\footnotetext{
${ }^{4}$ In particular, Metzler (1941) discusses the effects of several simple linear expectation formation rules on the dynamics of the business cycle.

5 See, e.g. Simon (1955), Kahnemann et al. (1986) or Smith (1991).
} 
agents seem to favour forecasting rules that did well in the past 6

Based on this empirical evidence, we want to allow producers to choose between two different predictors in order to determine expected future sales. In particular, producers of type one form extrapolative extrapolations, i.e. they extrapolate the trend in consumption in order to estimate their future sales. For instance, if consumption has decreased (increased) in the recent past, trend-extrapolating producers believe that consumption will fall (rise) further. Technically, this type of expectation formation can be described by

$$
U_{t}^{E}=C_{t-1}+c\left(C_{t-1}-C_{t-2}\right),
$$

where $c>0$ simply represents a positive extrapolation parameter.

Producers of the second type form regressive expectations, i.e. they believe that consumption will always return to its equilibrium value, $\bar{C}$, in the long run. Thus, the regressive predictor may be expressed as

$$
U_{t}^{R}=C_{t-1}+f\left(\bar{C}-C_{t-1}\right),
$$

where $0<f<1$ stands for the expected adjustment speed towards equilibrium.

Let $w_{t}^{E}$ and $w_{t}^{R}$ be the fractions of producers who respectively choose the extrapolative and the regressive predictor. Overall, expected future sales of consumption goods, $U_{t}$, may then be expressed as a weighted average of both type of expectations 7 i.e.

$$
U_{t}=w_{t}^{E} U_{t}^{E}+w_{t}^{R} U_{t}^{R} .
$$

We now want to specify how producers choose a certain forecasting rule in order to predict future sales, i.e. how fractions $w_{t}^{E}$ and $w_{t}^{R}$ evolve over time. A quite natural approach is that producers tend to select forecasting rules with a high evolutionary fitness 8 The better one predictor performed in the past, the more attractive this rule will be and the more producers will choose it. In our case, producers shall favour forecasting rules that produce low prediction errors. In this sense, the attractiveness or fitness of both predictors can respectively be written

\footnotetext{
${ }^{6}$ Further evidence on dynamic predictor selection and heterogeneous expectation formation in different environments is provided by Reitz and Westerhoff (2003), Alfarano et al. (2005), Boswijk et al. (2007) and Goldbaum and Mizrach (2008).

7 Anufriev et al. (2008) argue that the most natural way to embed heterogeneous expectations into a linear macroeconomic model that has no explicit microfoundation is to use a weighted average of individual expectations. However, we will discuss this issue in more detail in appendix A.

8 This idea was put forward by Brock and Hommes (1997, 1998).
} 
as

$$
\begin{aligned}
& A_{t}^{E}=-\left(C_{t-1}-U_{t-1}^{E}\right)^{2}+m A_{t-1}^{E}, \\
& A_{t}^{R}=-\left(C_{t-1}-U_{t-1}^{R}\right)^{2}+m A_{t-1}^{R}-\mu .
\end{aligned}
$$

The first terms of (10) and (11) simply describe the last period's squared forecasting errors, while the second terms reflect producers' memory. The associated memory parameter, $m$, is restricted to $0 \leq m \leq 1$. For instance, if $m=0$, producers have no memory at all and the attractiveness of a certain predictor does not depend on past forecasting errors. But the larger producers' memory, the more a certain strategy's attractiveness will also be subject to its past performance. Finally, for $m=1$, producers will have a perfect memory and the attractiveness of a certain predictor consequently incorporates all past forecasting errors. Also note that regressive expectations may be regarded as a quite sophisticated predictor. In order to determine equilibrium national income, $\bar{Y}$, and the adjustment speed, $f$, producers may have to evaluate various macroeconomic variables. In other words, forming regressive expectations requires more effort than forming extrapolative expectations. Thus, regressive expectations are relatively more expensive. In (11), these higher information gathering costs are captured by the positive term $\mu \geq 0$. Moreover, Branch (2004) argues that $\mu$ may also be interpreted as a predisposition effect. In this sense, $\mu>0$ means that agents have a behavioural preference for simple extrapolative expectations.

The evolution of the fractions, $w_{t}^{E}$ and $w_{t}^{R}$ is based on a discrete choice approach (see, e.g. Manski and McFadden, 1981), i.e.

$$
w_{t}^{E}=(1-\delta) \frac{\exp \left[g A_{t}^{E}\right]}{\exp \left[g A_{t}^{E}\right]+\exp \left[g A_{t}^{R}\right]}+\delta w_{t-1}^{E}
$$

and

$$
w_{t}^{R}=(1-\delta) \frac{\exp \left[g A_{t}^{R}\right]}{\exp \left[g A_{t}^{E}\right]+\exp \left[g A_{t}^{R}\right]}+\delta w_{t-1}^{R} .
$$

Note that (12) and (13) incorporate the more general case of asynchronous updating, i.e. in each time step only a fraction $(1-\delta)$ of producers reconsiders its decision for a certain predictor 9 Obviously, $\delta$ is restricted to $0 \leq \delta \leq 1$. Moreover, parameter $g \geq 0$ is the well-known intensity of choice, and simply reflects how quickly producers switch from one forecasting rule to another. For $g=0$ there is no switching

${ }^{9}$ For (financial market) models with asynchronous updating, see Diks and van der Weide (2005) and Hommes et al. (2005a). For an interesting alternative specification, see also Dieci et al. (2006). 
at all, whereas in the extreme case of $g=\infty$ producers react to even minimal differences in the attractiveness of both predictors. In such a case, a fraction of $(1-\delta)$ producers will instantly switch to the best forecasting rule. In other words: the switching intensity increases with parameter $g$.

Combining (11)-(13) now reveals that our model corresponds to a higherorder nonlinear difference equation. By introducing the auxiliary variables $X_{t}=Y_{t-1}$ and $Z_{t}=X_{t-1}=Y_{t-2}$, this difference equation may be extended to the following system of first-order nonlinear difference equations

$$
\begin{aligned}
Y_{t}= & b(1+k)\left(f X_{t-1}-X_{t-1}-f \bar{Y}\right) w_{t-1}^{R}+ \\
& b(1+k)\left(\tilde{\theta}_{2}-\delta \tilde{\theta}_{2}+\delta w_{t-1}^{R}\right)\left(f \bar{Y}+Y_{t-1}-f Y_{t-1}\right)+ \\
& b(1+k)\left(\tilde{\theta}_{1}-\delta \tilde{\theta}_{1}+\delta w_{t-1}^{E}\right)\left(Y_{t-1}+c Y_{t-1}-c X_{t-1}\right)- \\
& b(1+k)\left(X_{t-1}+c X_{t-1}-c Z_{t-1}\right) w_{t-1}^{E}+b Y_{t-1}+\bar{I}, \\
X_{t}= & Y_{t-1}, \\
Z_{t}= & X_{t-1} \\
w_{t}^{E}= & (1-\delta) \tilde{\theta}_{1}+\delta w_{t-1}^{E} \\
w_{t}^{R}= & (1-\delta) \tilde{\theta}_{2}+\delta w_{t-1}^{R}, \\
A_{t}^{E}= & -b^{2}\left(Y_{t-1}-X_{t-1}+c X_{t-1}+Z_{t-1}\right)^{2}+m A_{t-1}^{E}, \\
A_{t}^{R}= & -b^{2}\left(Y_{t-1}-X_{t-1}+f X_{t-1}-f \bar{Y}\right)^{2}+m A_{t-1}^{R}-\mu,
\end{aligned}
$$

where $\tilde{\theta}_{1}=\frac{\theta_{1}}{\theta_{1}+\theta_{2}}, \tilde{\theta}_{2}=\frac{\theta_{2}}{\theta_{1}+\theta_{2}}$ and

$$
\begin{aligned}
& \theta_{1}=\exp \left[g m A_{t-1}^{E}-g b^{2}\left(Y_{t-1}-X_{t-1}-c X_{t-1}+c Z_{t-1}\right)^{2}\right], \\
& \theta_{2}=\exp \left[g m A_{t-1}^{R}-g \mu-g b^{2}\left(Y_{t-1}-X_{t-1}+f X_{t-1}-f \bar{Y}\right)^{2}\right] .
\end{aligned}
$$

Using a combination of both analytical and numerical tools, we investigate the properties of this $7 \mathrm{D}$-nonlinear dynamical system in the subsequent three sections.

\section{Fixed point and local stability analysis}

Let us begin our analytical survey by noting that (11)-(梠) yield

$$
Y_{t}=(1+k)\left(U_{t}-U_{t-1}\right)+b Y_{t-1}+\bar{I} .
$$

It is easy to see from this equation that the model has the unique fixed point

$$
\bar{Y}=\frac{\bar{I}}{1-b},
$$


that corresponds to the well-known Keynesian multiplier solution. Similar calculations reveal that the other steady state variables of system (14)-(20) are determined by

$$
\begin{aligned}
\bar{X} & =\bar{Y}, \\
\bar{Z} & =\bar{Y}, \\
\overline{A^{R}} & =\frac{\mu}{m-1}, \\
\overline{A^{E}} & =0, \\
\overline{w^{R}} & =\frac{\theta}{1+\theta}, \\
\overline{w^{E}} & =\frac{1}{1+\theta},
\end{aligned}
$$

where $\theta=\exp \left[\frac{g \mu}{m-1}\right]$.

By using these results, we are able to linearise our nonlinear system around this unique steady state, and obtain the following Jacobian

$$
J=\left(\begin{array}{ccc}
a_{11} & \ldots & a_{17} \\
\vdots & \ddots & \vdots \\
a_{71} & \ldots & a_{77}
\end{array}\right),
$$

where the coefficients $a_{i j}(i, j=1,2, \ldots, 7)$ denote the partial derivatives of system (14)-(201) calculated at the above defined steady state.

Our fixed point is then locally asymptotically stable if and only if all seven eigenvalues of the above Jacobian, $J$, are less than one in absolute value 10 Straightforward computations reveal that in our case the first four eigenvalues are given by $\lambda_{1,2}=m$ and $\lambda_{3,4}=\delta$ and are thus by definition always less than one in absolute value. In turn, the remaining three eigenvalues, $\lambda_{5}, \lambda_{6}$ and $\lambda_{7}$, are determined by the roots of the characteristic polynomial

$$
P(\lambda)=\lambda^{3}+a_{1} \lambda^{2}+a_{2} \lambda+a_{3}=0,
$$

with the coefficients

$$
\begin{aligned}
& a_{1}=-\frac{b(2+c+c k+k-\theta(f-2+(f-1) k))}{(1+\theta)}, \\
& a_{2}=\frac{b(1+k)(1+2 c+\theta(1-f))}{1+\theta}
\end{aligned}
$$

\footnotetext{
10 See, e.g. Galor (2007) and, for a more comprehensive survey on stability conditions for higher-order linear systems, also Gandolfo (2009).
} 
and

$$
a_{3}=-\frac{b c(1+k)}{1+\theta} .
$$

Unfortunately, this polynomial is too complex to derive the remaining three eigenvalues explicitly. However, it can be shown that all eigenvalues of the above given characteristic polynomial are less than one in absolute value if and only if the following conditions simultaneously apply 11

$$
\begin{array}{r}
1+a_{1}+a_{2}+a_{3}>0 \\
1-a_{1}+a_{2}-a_{3}>0 \\
1-a_{2}+a_{1} a_{3}-a_{3}^{2}>0 \\
3+a_{1}-a_{2}-3 a_{3}>0 .
\end{array}
$$

The first three conditions are thereby associated with different types of bifurcation: violation of (31) is a necessary condition for a flip bifurcation, violation of (32) is a necessary condition for a saddle-node bifurcation and violation of (33) is a necessary condition for a NeimarkSacker bifurcation to occur. Moreover, these three conditions may also be regarded as the crucial conditions for smooth stability, i.e. if we have a stable fixed point and then continuously change the parameter under control, one of the first three conditions will always be violated before condition (34) is hit 12

Straightforward calculations now reveal that for our model conditions (31)-(34) yield

$$
\begin{aligned}
& 1-b>0 \\
& 1+3 b-2 b f+2 b k-2 b f k+\frac{2 b(2 c+f)(1+k)}{1+\theta}>0 \\
& 1-\frac{b+2 b c(1-b)}{(1+\theta)^{2}}-\frac{b k(1+2 c)}{(1+\theta)^{2}}+\frac{b^{2} c k(3+k)}{(1+\theta)^{2}}- \frac{b \theta(3+2 c)}{(1+\theta)^{2}}+\frac{2 b \theta(b c+f)}{(1+\theta)^{2}}-\frac{b k \theta(3+2 c)}{(1+\theta)^{2}}+ \\
& \frac{b^{2} c k \theta(3-2 f)}{(1+\theta)^{2}}+\frac{b f \theta(2 k-b c)}{(1+\theta)^{2}}+\frac{b^{2} c k^{2} \theta(1-f)}{(1+\theta)^{2}}>0 \\
& 3-3 b-2 b k+\frac{2 b f(1+k)}{1+\theta}>0
\end{aligned}
$$

\footnotetext{
11 See, e.g. Lines (2007). For an alternative set of stability conditions see, Chiarella and $\mathrm{He}(2005)$.

12 See, e.g. Lines (2007), who proves that no parameter combination satisfies the last condition as an equality while simultaneously satisfying the first three conditions.
} 
Taking into account that $0<b<1$ and $0<f<1$, it is not difficult to see that the first two conditions are always fulfilled. In turn, the last two conditions may be violated. In particular, condition (37) and its possible violation for certain parameter combinations thereby indicate that under a smooth change of parameters our unique fixed point may only loose its stability via a Neimark-Sacker bifurcation. In the following sections we attempt to scrutinise these interesting analytical findings by giving some numerical examples of the dynamics our model may produce.

\section{A numerical example of the dynamics}

We start our numerical analysis with a brief illustration of the dynamics our model may produce. For this purpose, we choose the following parameter setting: $b=0.75, c=1, f=0.6, g=250, k=0.1, m=0.05$, $\delta=0.15$ and $\mu=0.01$. Furthermore, investments are fixed at $\bar{I}=10$, implying that equilibrium income is then given by $\bar{Y}=40$.

[Figure 1 about here.]

Figure 1 now displays a typical simulation run for the above given parameter setting. The panels in the first two rows depict the evolution of national income, consumption, expected sales and inventory production over a period of 100 time steps. A longer transient of 10000 steps is omitted. Obviously, our model is able to produce intricate and irregular business cycles with booms and slumps. Both the frequency and amplitude of the cycles vary over time. Please note that these upswings and downturns of national income are created endogenously within our model. No exogenous shocks are necessary to explain or maintain the persisting cyclical motions of national income.

Instead, dynamics may be explained as follows. The bottom panels of figure 1 clearly indicate that in many time steps almost all agents either form regressive or extrapolative expectations. Let us suppose that all producers use the regressive forecasting rule, i.e. $w_{t}^{R}=1$ and $w_{t}^{E}=0$. In such a case, our model is reduced to the following second-order linear difference equation

$$
Y_{t}=b(2+k-f-f k) Y_{t-1}+b(f-1)(1+k) Y_{t-2}+\bar{I},
$$

the fixed point of which is given by the previously presented Keynesian multiplier solution.

Straightforward calculations reveal that the fixed point is (globally asymptotically) stable for our initial parameter setting 13 Hence, pe-

\footnotetext{
${ }^{13}$ For a comprehensive survey on the stability of linear difference equations, see, e.g. Gandolfo (2009).
} 
riods in which (almost) all producers form regressive expectations tend to have a stabilising impact on dynamics.

If, in turn, all producers form extrapolative expectations, the evolution of national income is described by

$$
\begin{aligned}
Y_{t}= & b(2+c+k+c k) Y_{t-1}-(1+k)(1+2 c) Y_{t-2}+ \\
& c(1+k) Y_{t-3}+\bar{I}
\end{aligned}
$$

a third-order linear difference equation. Again, the fixed point of (40) corresponds to the Keynesian multiplier solution. But this time the fixed point is unstable within our parameter setting. Hence, our model behaves in an unstable manner if all producers rely on extrapolative expectations and extrapolative expectations may be regarded as a destabilising force 14

However, the panels presented in figure 1 clearly indicate that output neither converges to its fixed point nor does it explode, and that neither regressive nor extrapolative expectations will permanently dominate dynamics. It rather seems to be the interplay between these two competing forces that leads to intricate endogenous business cycles within our model. This interplay may be explained as follows. Let us suppose that national income and consumption fluctuate closely above their equilibrium values. In such a case, the forecasting errors of both predictors become quite small. Accordingly, both predictors will yield similar results. But since the use of regressive expectations is relatively more expensive, extrapolative expectations appear more attractive. Thus, a large fraction of producers will immediately switch to extrapolative expectations, and regressive expectations are increasingly crowded out of the market. As a result, national income will be driven away from its equilibrium in an oscillatory fashion.

Nonetheless this motion will not last forever. At some point in time, extrapolative expectations may produce quite strong prediction errors, and the benefits of forming regressive expectations may now outweigh the higher information gathering costs and the predisposition effect. Since regressive expectations now appear superior, more and more producers will switch to regressive expectations and a period of convergence sets in. Producers decrease both production of consumption goods for sales and inventory purposes and, as a result, national income, $Y$, will start to fall. This decrease continues until national income is close to its equilibrium value again and the pattern may repeat itself.

\footnotetext{
${ }^{14}$ In the next section it will become clear that these claims may not be generalised too much. For some parameter combinations stability may in fact crucially depend on the interplay between parameters $c$ and $f$, i.e. the two parameters defining the expectational forces in our model.
} 
Note also that in an upswing, producers become optimistic and thus increase their inventory. Accordingly, production is higher than in a model without any inventory stock production. In contrast, in an economic downswing, firms deplete their inventory, which has a negative impact on production. Overall, we conclude that not only the expectation formation of boundedly rational heterogeneous producers but also their inventory management may in fact play an important role in economic activity 15

\section{Routes to endogenous business cycles}

In the previous section, we only analysed the dynamics for a particular parameter setting. But since the true parameters of the economy are unknown, we would like to broaden our view in this section by exploring the model's dynamical behaviour for a wider range of parameter combinations.

[Figure 2 about here.]

Let us begin our numerical investigation with an illustration of the effects of parameters $f$ and $c$, the corresponding bifurcation diagrams of which are depicted in figure 2 Hereby, each parameter is increased in 500 discrete steps, as indicated on the axis, while all other parameters are identical to those in our default parameter setting used in the previous sections. The first bifurcation diagram suggests that the stability of the fixed is independent of the expected adjustment speed towards equilibrium income. For instance, when $f$ is too low or too high, an explosion of the dynamics (represented by the blank areas in the diagram) occurs, while for values of $f$ between 0.45 and 0.65 , intricate endogenous dynamics in the form of business cycles with varying amplitudes arise. Put differently, for our default parameter setting we will not observe a convergence towards the model's unique fixed point for any value of $0<f<1$.

In turn, the second bifurcation diagram for parameter $c$ exhibits that national income converges towards its equilibrium when $c$ is low. But when $c$ exceeds a certain threshold, dynamics change essentially. The fixed point suddenly loses its local asymptotic stability, and pronounced endogenous dynamics set in. This threshold may be calculated by the

\footnotetext{
15 As it turns out, in rare situations the inventory becomes negative for our initial parameter setting. This issue may be circumvented by either adding a fixed buffer or an inventory floor to (4). However, such a fixed buffer does not affect the model's law of motion. For a more detailed description of a model with an inventory floor, see, e.g. Sushko et al. (2009).
} 
help of the stability conditions presented in the previous section. For our particular parameter setting it is $c=0.5905$. Moreover, our analytical results also indicate that with respect to a smooth increase in parameter $c$, our fixed point may only lose it stability via a Neimark-Sacker bifurcation. However, the sharp and sudden change in the dynamical behaviour of the system now suggests that our Neimark-Sacker bifurcation is of subcritical type and is furthermore accompanied by a so-called Chenciner or crater bifurcation 16

To be precise, a Chenciner bifurcation is a generic phenomenon that may occur in higher dimensional nonlinear systems with two or more parameters. Due to the Chenciner bifurcation, our stable fixed point coexists with two limit cycles. The first limit cycle is unstable and repelling, and defines the fixed point's basin of attraction, i.e. all trajectories starting within that region in parameter space will finally converge to our locally asymptotically stable fixed point. Instead, trajectories starting outside that neighbourhood of the fixed point are attracted by the second and stable limit cycle. Similarly to the occurrences in the case of a pure subcritical Neimark-Sacker bifurcation, the basin of attraction eventually decreases and shrinks as parameter $c$ approaches its critical bifurcation value. At the critical bifurcation value, the unstable limit cycle finally merges with the fixed point, such that the fixed point becomes unstable. However, figure 3 clearly indicates that we may observe a global bifurcation before the critical bifurcation value is reached. Depending on initial conditions trajectories may already start outside the shrinking basin of attraction and are thus immediately attracted by the remaining stable limit cycle. As a consequence, we will then observe a sharp, sudden change in dynamics 17

[Figure 3 about here.]

It is a well-known fact in the economic literature that such a coexistence of attractors may cause hysteresis phenomena, and may thus have important practical economic implications 18 For instance, consider a situation in which the crucial parameter $c$ changes due to some exterior effects such that it crosses its critical bifurcation value and the fixed point destabilises through the above-described Chenciner bifurcation scenario. Once the fixed point has lost its stability, all trajectories will

\footnotetext{
${ }^{16}$ For a complete mathematical treatment of the Chenciner bifurcation, see Kuznetsov (2004). Moreover, economic applications covering this kind of bifurcation are given by Gaunersdorfer et al. (2008), Lines and Westerhoff (2009), Neugart and Tuinstra (2003), Kind (1999), Agliari (2006) and Agliari et al. (2005).

17 In contrast, in the case of a pure subcritical Neimark-Sacker bifurcation, the fixed point becomes unstable and all trajectories simply diverge.

18 See, e.g. Agliari (2006)or Agliari et al. (2006).
} 
be attracted by the remaining stable limit cycle. Since this attracting limit cycle is now relatively far away from the fixed point, all trajectories are also clearly outside the fixed point's basin of attraction. As a consequence, a simple restoration of the previously stable parameter value is not enough to allow the system to return to its equilibrium ever again. In other words: any stabilisation policy aimed at a mere change of parameters must clearly fail unless the trajectories are not pushed back into the (attracting) vicinity of the steady state.

Another interesting numerical phenomenon can be observed at about $c=0.70$. For values of $c$ close before this threshold all trajectories are attracted by the stable limit cycle. At about $c=0.70$, however, dynamics explode, while for values above $c=0.70$ a strange attractor emerges, the amplitude of which is larger than that of the stable limit cycle. Even a slight increase in parameter $c$ may thus have a significant effect on dynamics.

From an economic point of view, such a destabilising effect of an increase in parameter $c$ may be comprehended as follows. Recall that parameter $c$ denotes the expected speed of deviation from equilibrium income. The higher $c$ is, the more optimistic or pessimistic producers are, and the stronger these producers will extrapolate the trend in consumption, which apparently tends to amplify dynamics. Furthermore, our bifurcation diagram for parameter $c$ indicates that the amplitude of the business cycle increases with $c$.

[Figure 4 about here.]

Nevertheless, trend-extrapolating producers may not be regarded as a source of instability per se since such a destabilising effect of parameter $c$ has to be treated carefully. In fact, stability depends on the balance between parameters $c$ and $f$. To understand this, please take into account that our crucial stability condition (33) implicitly defines a function in $(c, f)$-parameter space separating stable from unstable parameter combinations 19 The upper panel of figure 4 now illustrates these stable (blank) and unstable (black) regions in parameter space for our default parameter setting. Clearly, within our default parameter setting the effects of a variation of parameters $c$ and $f$ are unambiguous, i.e. an increase in parameter $c$ always tends to have a destabilising impact on dynamics, and a single variation of parameter $f$ does not affect stability at all. But these results have to be treated more carefully and may not be generalised to other parameter settings. The lower panel of figure 4 gives a stylised sketch of the stable and unstable regions in $(c, f)$ parameter space. This sketch may be regarded to hold for a broader

\footnotetext{
19 Please recall, that the other stability condition which may be violated is completely independent of parameter $c$.
} 
range of parameters settings. Let us now assume that we have a stable parameter setting in the upper left blank region. A single increase in parameter $c$, i.e. a rightward movement into the black unstable region of parameter combinations, will then clearly have a destabilising impact on dynamics. The same holds true for a single decrease in parameter $f$, i.e. a downward movement in the diagram. In turn, an increase in parameter $c$ together with a simultaneous decrease in parameter $f$, i.e. a right-downward movement in parameter space, may have a destabilising impact on dynamics in the first place, but any further parameter shift into the same direction will then at some point of time clearly stabilise dynamics again.

[Figure 5 about here.]

Figure 5 now continues with two bifurcation diagrams for parameter $g$, which are plotted for slightly different initial conditions for national income. Obviously, the result of this exercise exhibits certain similarities to our previous bifurcation diagram for parameter $c$. Visual inspection reveals that when the intensity of choice is low, national income, $Y$, converges towards its equilibrium value and no fluctuations in business cycles appear. But as parameter $g$ increases and finally exceeds a certain threshold of about $g=75.477$, the dynamical behaviour of our system will change fundamentally again, i.e. the fixed point abruptly loses its stability, and business cycles with substantial amplitude are created endogenously within our model. The bifurcation diagrams presented on the right-hand side of figure 5 clearly displays that we may also observe a global bifurcation before the critical bifurcation value is reached. Again depending on initial conditions all trajectories may already start outside the fixed point's basin of attraction and may thus immediately converge to some coexisting strange attractor. The latter point is furthermore illustrated by the phase space plot depicted in figure 6

[Figure 6 about here.]

All in all, we thus conclude that, equivalently to our previous case for parameter $c$, a Chenciner bifurcation also takes place for parameter $g$ exceeding a certain threshold.

But why does an increase in parameter $g$ lead to complex endogenous dynamics? Recall that parameter $g$ denotes producers' intensity of choice, and that the higher $g$ is, the more sensitively producers will react to even the slightest differences in the attractiveness of both predictors. Hence, the higher $g$ is, the faster producers will switch from one 
forecasting rule to another. Apparently, such behaviour tends to have a destabilising impact on dynamics 20

[Figure 7 about here.]

Finally, the bifurcation diagrams for parameters $b$ and $k$ are depicted in figure 7 . They lead to the following results. When parameter $b$ is low, national income converges to its equilibrium value. However, when $b$ exceeds a certain threshold, the fixed point will lose its stability, and both complex and explosive dynamics may occur. Similarly, the bifurcation diagram for parameter $k$ reveals that intricate endogenous dynamics but also unstable trajectories may appear. Furthermore, both bifurcation diagrams indicate that the amplitude of business cycles seems to increase with both parameters. It follows from this that an increase in one of these parameters has a destabilising effect on dynamics.

At least with respect to an increase in parameter $k$, this result is economically quite clear. Recall that the higher $k$ is, the more inventory producers wish to hold. Hence, an increase in parameter $k$ implies that inventory fluctuations will become more dominant, and as a consequence, we will observe stronger fluctuations in both inventory and national income. Similar arguments also hold for parameter $b$, though the effective mechanism at work seems to be more complicated. First of all, an increase in parameter $b$ will lead to an increase in equilibrium national income and consumption via the multiplier. But since producers forming regressive sales expectations directly base their decision on the level of equilibrium consumption, such an increase will also boost overall production of consumption goods. This, in turn, urges producers to expand their inventory, and thus tends to have the above-described destabilising effect on dynamics.

As a concluding remark, we wish to point out that all bifurcation diagrams presented in this section verify that our nonlinear model in contrast to Metzler's original linear approach - is able to produce intricate endogenous dynamics for a broad range of parameters.

\section{Conclusions}

In this paper, we extend Metzler's (1941) well-known linear business cycle model by developing a framework in which producers can either apply a simple and cheap extrapolative or a more sophisticated but relatively more costly regressive predictor to determine expected

${ }^{20}$ For similar results in different frameworks, see, e.g. Brock and Hommes (1997), Brock et al. (2006) or Westerhoff (2006). 
future sales. Producers thereby display a boundedly rational learning behaviour, in the sense that they tend to select forecasting rules with a high evolutionary fitness. As it turns out, the motion of national income is then described by a higher-order nonlinear difference equation, which has a unique fixed point. Using a mixture of analytical and numerical tools, we try to describe the characteristics of the model's dynamical system and obtain the following results.

With respect to a smooth increase of the control parameters the unique fixed point may only lose its local asymptotic stability via a subcritical Neimarck-Sacker bifurcation, which may furthermore be accompanied by a so-called Chenciner bifurcation. Once the fixed point has destabilised, complex business cycles with varying amplitude are created endogenously within our model for a broad range of parameter combinations. In particular, no exogenous shocks are necessary to explain or maintain the persisting up- and downward movements of national income and related variables, such as consumption or inventory stock production. Instead, dynamics are driven by the interplay between the different expectations. Our results thereby indicate that regressive expectations may be seen as a stabilising force whereas extrapolative expectations tend to have a destabilising impact on dynamics. Moreover, the crucial factor between these two competing forces seems to be producers' intensity of choice. Since both our analytical and numerical results suggest that an increase in producers' intensity of choice may lead to complex dynamics, we also conclude that producers, who quickly switch between predictors may be regarded as a source of instability.

\section{Appendix}

\section{A. Aggregation of sales expectations}

In this appendix, we illustrate the conditions under which expected sales of consumption goods, $U_{t}$, may be expressed as a weighted average of both types of expectations. For the sake of simplicity, we restrict our analysis to the simplest case in which producers have no memory at all and have no preference for simple linear heuristics, i.e. $m=0$ and $\mu=0$. Given this, (10) and (11) will reduce to

$$
\begin{aligned}
& A_{t}^{E}=-\left(C_{t-1}-U_{t-1}^{E}\right)^{2}, \\
& A_{t}^{R}=-\left(C_{t-1}-U_{t-1}^{R}\right)^{2} .
\end{aligned}
$$

Moreover, we want to omit all cases of asynchronous updating. Hence, the fractions of agents using either the extrapolative or the regressive 
forecasting rule are respectively denoted by

$$
w_{t}^{E}=\frac{\exp \left[g A_{t}^{E}\right]}{\exp \left[g A_{t}^{E}\right]+\exp \left[g A_{t}^{R}\right]}
$$

and

$$
w_{t}^{R}=\frac{\exp \left[g A_{t}^{R}\right]}{\exp \left[g A_{t}^{E}\right]+\exp \left[g A_{t}^{R}\right]} .
$$

Let us now suppose that there are $N$ firms in the market. All firms shall be equal in size in the sense that each firm is able to sell the same amount of consumption goods. Nevertheless, each firm still forms its individual expectations about its future sales, which are now specified by

$$
\begin{aligned}
U_{i, t}^{E} & =\frac{C_{t-1}}{N}+c\left(\frac{C_{t-1}}{N}-\frac{C_{t-2}}{N}\right) \\
& =\frac{1}{N}\left(C_{t-1}+c\left(C_{t-1}-C_{t-2}\right)\right) \\
& =\frac{1}{N} U_{t}^{E}
\end{aligned}
$$

and

$$
\begin{aligned}
U_{i, t}^{R} & =\frac{C_{t-1}}{N}+f\left(\frac{\bar{C}}{N}-\frac{C_{t-1}}{N}\right) \\
& =\frac{1}{N}\left(C_{t-1}+f\left(\bar{C}-C_{t-1}\right)\right) \\
& =\frac{1}{N} U_{t}^{R} .
\end{aligned}
$$

Accordingly, the individual performance of each predictor may be written as

$$
\begin{aligned}
A_{i, t}^{E} & =-\left(\frac{C_{t-1}}{N}-U_{i, t-1}^{E}\right)^{2} \\
& =-\left(\frac{C_{t-1}}{N}-\frac{U_{t-1}^{E}}{N}\right)^{2} \\
& =\frac{1}{N^{2}} A_{t}^{E}
\end{aligned}
$$


and

$$
\begin{aligned}
A_{i, t}^{R} & =-\left(\frac{C_{t-1}}{N}-U_{i, t-1}^{R}\right)^{2} \\
& =-\left(\frac{C_{t-1}}{N}-\frac{U_{t-1}^{R}}{N}\right)^{2} \\
& =\frac{1}{N^{2}} A_{t}^{R} .
\end{aligned}
$$

The individual weighting function

$$
w_{i, t}^{E}=\frac{\exp \left[g_{i} A_{i, t}^{E}\right]}{\exp \left[g_{i} A_{i, t}^{E}\right]+\exp \left[g_{i} A_{i, t}^{R}\right]}
$$

may now be interpreted as the (individual) probability with which a particular firm chooses the extrapolative forecasting rule. Hence, expected future sales of a particular firm are determined by the following probability function

$$
U_{i, t}= \begin{cases}U_{i, t}^{E} & \text { with probability } w_{i, t}^{E} \\ U_{i, t}^{R} & \text { with probability } 1-w_{i, t}^{E}\end{cases}
$$

while overall expected sales are simply given by

$$
U_{t}=\sum_{i=1}^{N} U_{i, t} .
$$

If we furthermore suppose that all producers react equally sensitive to differences in the performance of both predictors, we are able to scale parameter $g$ so that the intensity of choice of each producer is given by

$$
g=\frac{g_{i}}{N^{2}} .
$$

With this simple transformation, it is now straightforward to see that our individual probability function (55) corresponds to the weighting function (41). Thus, for a sufficiently large number of firms, approximately $w_{t}^{E} N$ firms will choose the extrapolative predictor, while approximately $\left(1-w_{t}^{E}\right) N$ firms will select the regressive forecasting rule. Accordingly, the aggregate of expected sales of consumption goods may properly be approximated by

$$
U_{t}=w_{t}^{E} U_{t}^{E}+w_{t}^{R} U_{t}^{R} .
$$




\section{Acknowledgements}

This paper was presented at the 6th International Conference on Nonlinear Economic Dynamics, Jönköping, Sweden, June 2009. We thank the participants, in particular Jan Tuinstra, for their very helpful and encouraging comments.

We also wish to thank two anonymous referees, whose remarks helped us to considerably improve our paper.

\section{References}

Agliari, A., 2006. Homoclinic connections and subcritical Neimark bifurcation in a duopoly model with adaptively adjusted productions. Chaos, Solitons \& Fractals 29 (3), 739-755.

Agliari, A., Bischi, G.-I., Gardini, L., 2006. Some methods for the global analysis of closed invariant curves in two-dimensional maps. In: Puu, T., Sushko, I. (Eds.), Business Cycle Dynamics: Models and Tools. Springer, pp. 7-49.

Agliari, A., Gardini, L., Puu, T., 2005. Some global bifurcations related to the appearance of closed invariant curves. Mathematics and Computers in Simulation 68 (3), 201-219.

Akerlof, G., Shiller, R., 2009. Animal spirits: how human psychology drives the economy and why it matters for global capitalism. Princeton University Press, Princeton.

Alfarano, S., Lux, T., Wagner, F., 2005. Estimation of agent-based models: The case of an asymmetric herding model. Computational Economics 26 (1), 19-49.

Anufriev, M., Assenza, T., Hommes, C. H., Massaro, D., 2008b. Interest rate rules with heterogeneous expectations. CeNDEF Working Paper 08-08, University of Amsterdam.

Berardi, M., 2007. Heterogeneity and misspecifications in learning. Journal of Economic Dynamics and Control 31 (10), 3203-3227.

Blinder, A. S., Maccini, L. J., 1991. Taking stock: a critical assessment of recent research on inventories. The Journal of Economic Perspectives 5, 73-96.

Boswijk, H. P., Hommes, C. H., Manzan, S., 2007. Behavioral heterogeneity in stock prices. Journal of Economic Dynamics and Control 31 (6), 1938-1970.

Branch, W. A., 2004. The theory of rationally heterogeneous expectations: evidence from survey data on inflation expectations. Economic Journal 114 (497), 592-621.

Branch, W. A., McGough, B., 2010. Dynamic predictor selection in a new Keynesian model with heterogeneous expectations. Working Paper.

Branch, W. A., McGough, B., 2009. A new Keynesian model with heterogeneous expectations. Journal of Economic Dynamics and Control 33 (5), 1036-1051.

Brock, W. A., Dindo, P. D. E., Hommes, C. H., 2005. Adaptive rational equilibrium with forward looking agents. CeNDEF Working Paper 05-15, University of Amsterdam.

Brock, W. A., Hommes, C. H., 1997. A rational route to randomness. Econometrica 65 (5), 1059-1095.

Brock, W. A., Hommes, C. H., 1998. Heterogeneous beliefs and routes to chaos in a simple asset pricing model. Journal of Economic Dynamics and Control 22 (8-9), $1235-1274$. 
Chiarella, C., He, X., 2003. Dynamics of belief and learning under $a_{l}$-processes - the heterogeneous case. Journal of Economic Dynamics and Control 27, 503-531.

Dieci, R., Foroni, I., Gardini, L., He, X., 2006. Market mood, adaptive beliefs and asset price dynamics. Chaos, Solitons \& Fractals 29 (3), 520-534.

Diks, C., van der Weide, R., 2005. Herding, a-synchronous updating and heterogeneity in memory in a CBS. Journal of Economic Dynamics and Control 29 (4), 741-763.

DeGrauwe, P., 2008. DSGE-modelling when agents are imperfectly informed. ECB Working Paper, European Central Bank.

Evans, G., Honkapohja, S., 2001. Learning and expectations in macroeconomics. Princeton University Press, Princeton.

Franke, R., 1996. A Metzlerian model of inventory growth cycles. Structural Change and Economic Dynamics 7 (2), 243-262.

Franke, R., Lux, T., 1993. Adaptive expectations and perfect foresight in a nonlinear Metzlerian model of the inventory cycle. Scandinavian Journal of Economics 95 (3), 355-63.

Galor, O., 2007. Discrete Dynamical Systems. Springer-Verlag, Berlin.

Gandolfo, G., 2009. Economic Dynamics - 4th Edition. Springer-Verlag, Berlin.

Gaunersdorfer, A., Hommes, C. H., Wagener, F. O. O., 2008. Bifurcation routes to volatility clustering under evolutionary learning. Journal of Economic Behavior \& Organization 67 (1), 27-47.

Goldbaum, D., Mizrach, B., 2008. Estimating the intensity of choice in a dynamic mutual fund allocation decision. Journal of Economic Dynamics and Control 32 (12), 3866-8876.

Heemeijer, P., Hommes, C., Sonnemans, J., Tuinstra, J., 2009. Price stability and volatility in markets with positive and negative expectations feedback: an experimental investigation. Journal of Economic Dynamics and Control 33 (5), $1052-1072$.

Hommes, C., Huang, H., Wang, D., 2005a. A robust rational route to randomness in a simple asset pricing model. Journal of Economic Dynamics and Control 29 (6), 1043-1072.

Hommes, C., Sonnemans, J., Tuinstra, J., van de Velden, H., 2005b. Coordination of expectations in asset pricing experiments. Review of Financial Studies 18 (3), 955-980.

Kahnemann, D., Slovic, P., Tversky, A., 1986. Judgment under uncertainty: heuristics and biases. Cambridge University Press, Cambridge.

Kind, C., 1999. Remarks on the economic interpretation of Hopf bifurcations. Economics Letters 62 (2), 147-154.

Kuznetsov, Y. A, 2004. Elements of applied bifurcation theory. Springer-Verlag, New York.

Lines, M., 2007. Practical tools for identifying dynamics in discrete systems. Working paper, Department of Statistics, University of Udine.

Lines, M., Westerhoff, F., 2006. Expectations and the multiplier-accelerator model. In: Puu, T., Sushko, I. (Eds.), Business Cycle Dynamics: Models and Tools. Springer, pp. $255-276$.

Lines, M., Westerhoff, F., 2010. Inflation expectations and macroeconomic dynamics: the case of rational versus extrapolative expectations. Journal of Economic Dynamics and Control 34 (2), 246-257.

Manski, C. F., McFadden, D. (Eds.), 1981. Structural Analysis of Discrete Data with Econometric Applications. The MIT Press, Cambridge, Massachusetts. 
Marcet, A., Nicolini, J. P., 2003. Recurrent hyperinflations and learning. American Economic Review 93 (5), 1476-1498.

Matsumoto, A., 1998. Non-linear structure of a Metzlerian inventory cycle model. Journal of Economic Behavior \& Organization 33 (3-4), 481-492.

Metzler, L. A., 1941. The nature and stability of inventory cycles. The Review of Economics and Statistics 23 (3), 113-129.

Neugart, M., Tuinstra, J., 2003. Endogenous fluctuations in the demand for education. Journal of Evolutionary Economics 13 (1), 29-51.

Ramey, V. A., West, K. D., 1997. Inventories. NBER Working Paper Series 6315, National Bureau of Economic Research.

Reitz, S., Westerhoff, F., 2003. Nonlinearities and cyclical behavior: the role of chartists and fundamentalists. Studies in Nonlinear Dynamics and Econometrics 7 (4).

Simon, H. A., 1955. A behavioral model of rational choice. Quarterly Journal of Economics 9, 99-118.

Smith, V., 1991. Papers in experimental economics. Cambridge University Press, Cambridge.

Sushko, I., Wegener, M., Westerhoff, F., Zaklan, G., 2009. Endogenous business cycle dynamics within the inventory model of Metzler: adding an inventory floor. Nonlinear Dynamics in Psychology and Life Sciences 13, 223-233.

Tuinstra, J., Wagener, F., 2007. On learning equilibria. Economic Theory 30 (3), 493-513.

Wegener, M., Westerhoff, F., Zaklan, G., 2009. A metzlerian business cycle model with nonlinear heterogeneous expectations. Economic Modelling 26, 715-720.

Westerhoff, F. H., 2006. Business cycles, heuristic expectation formation, and contracyclical policies. Journal of Public Economic Theory 8 (5), 821-838.

Westerhoff, F., Hohnisch, M., 2008. Business cycle synchronization in a simple Keynesian macro model with socially-transmitted economic sentiment and international sentiment spill-over. Structural Change and Economic Dynamics 19, 249-259. 

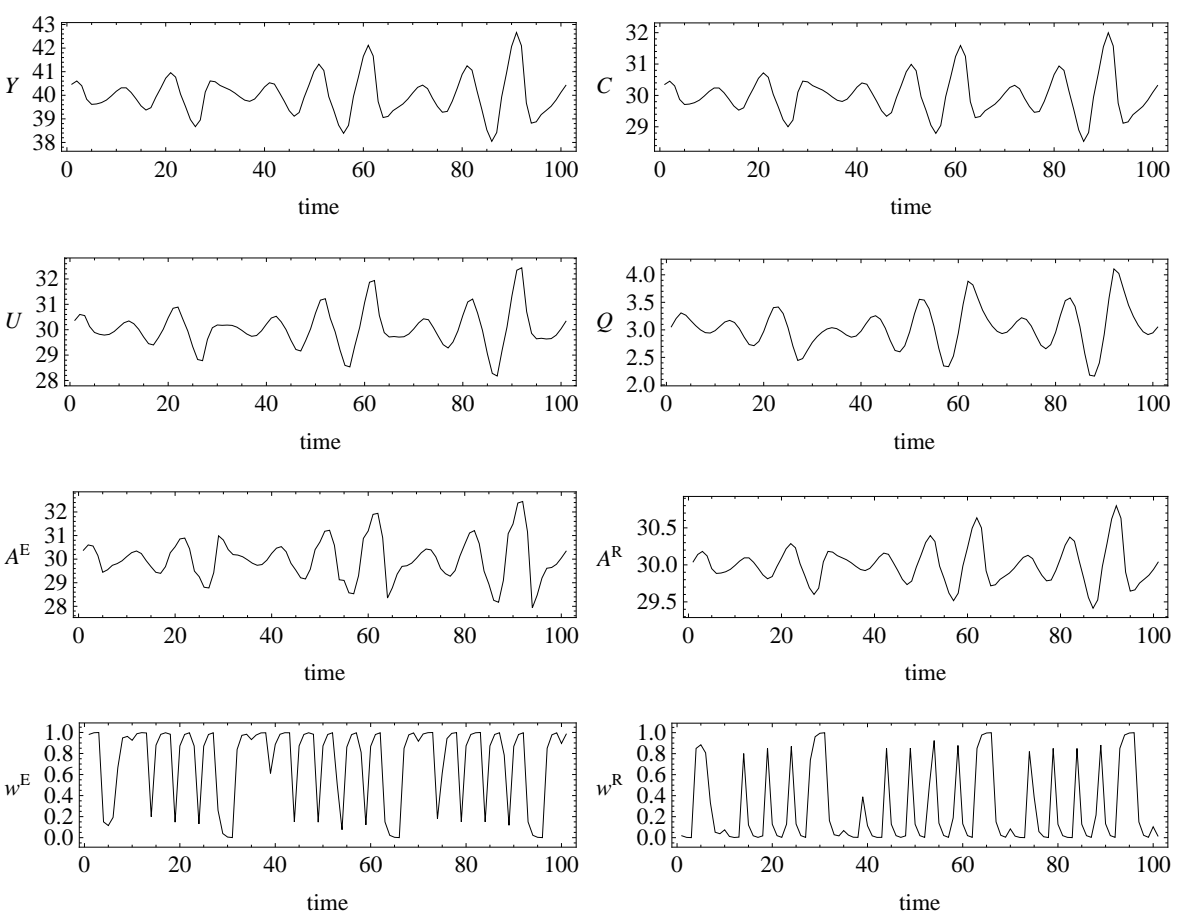

Figure 1. Evolution of national income $Y$ and related variables in a simulation run for $m=0.05, \mu=0.01, \delta=0.15, g=250, c=1, f=0.6, k=0.1$ and $b=0.75$. Equilibrium values of income and consumption are $\bar{Y}=40$ and $\bar{C}=30$. 

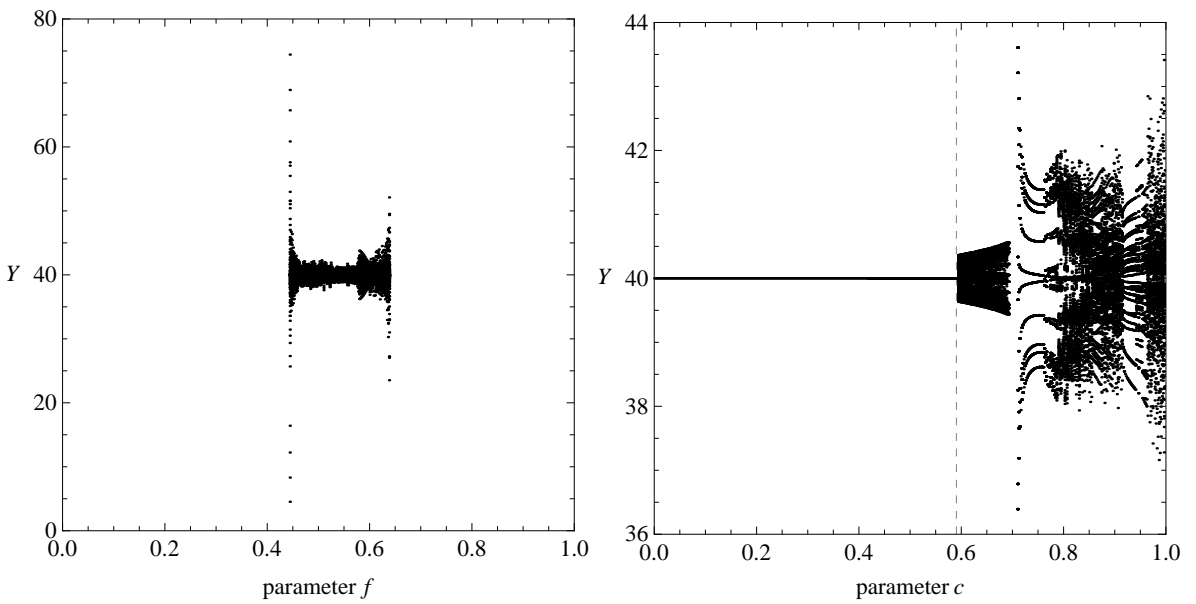

Figure 2. Bifurcation diagrams for parameters $f$ and $c$. Each parameter is increased in 500 discrete steps, as indicated on the axis. Dynamics are plotted after a transient of 5000 observations. The other parameters are identical to those in figure 1 The dashed line depicts the calculated critical bifurcation value for parameter $c$ 

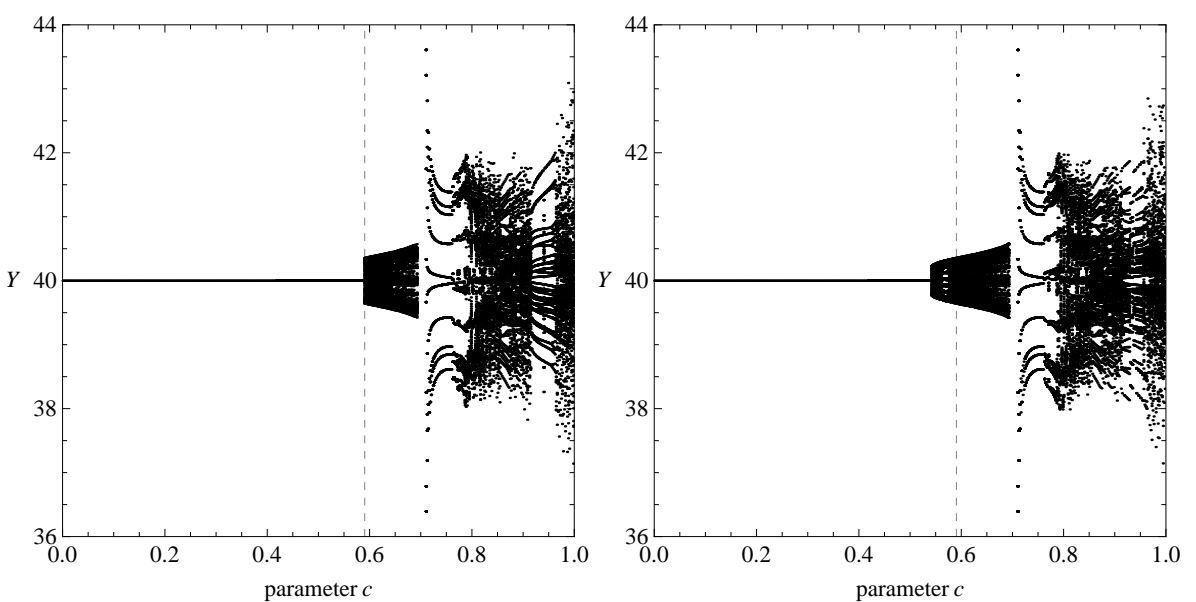

Figure 3. Bifurcation diagrams for parameter $c$ plotted with different starting values for national income. Left-hand side: $Y_{0}=40.01$. Right-hand side: $Y_{0}=40.1$. Depending on initial conditions, we may observe a global bifurcation before the critical bifurcation value (depicted by the dashed line) is reached. 

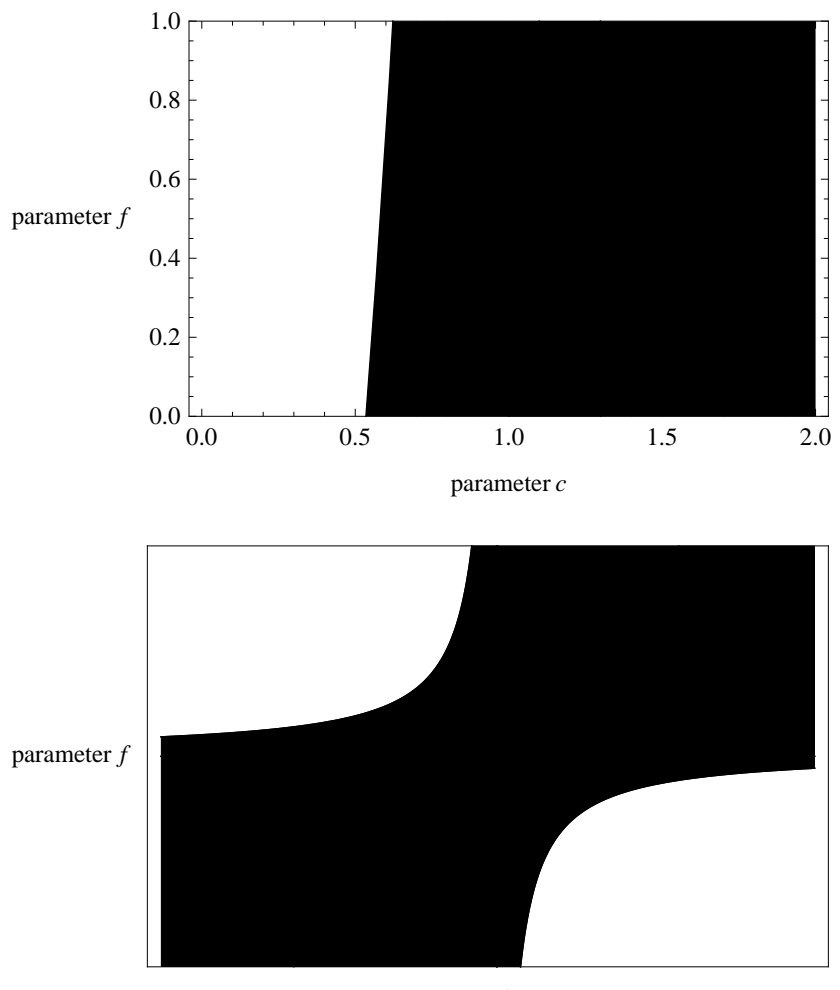

parameter $c$

Figure 4. Stability condition (33) implicitly defines a function in $(c, f)$-parameter space, which separates stable (blank) from unstable (black) parameter combinations. Upper panel: stable and unstable combinations of parameters $c$ and $f$ within or default parameter setting. Lower panel: stylised sketch of stable and unstable combinations which holds for a more general set of parameters. 

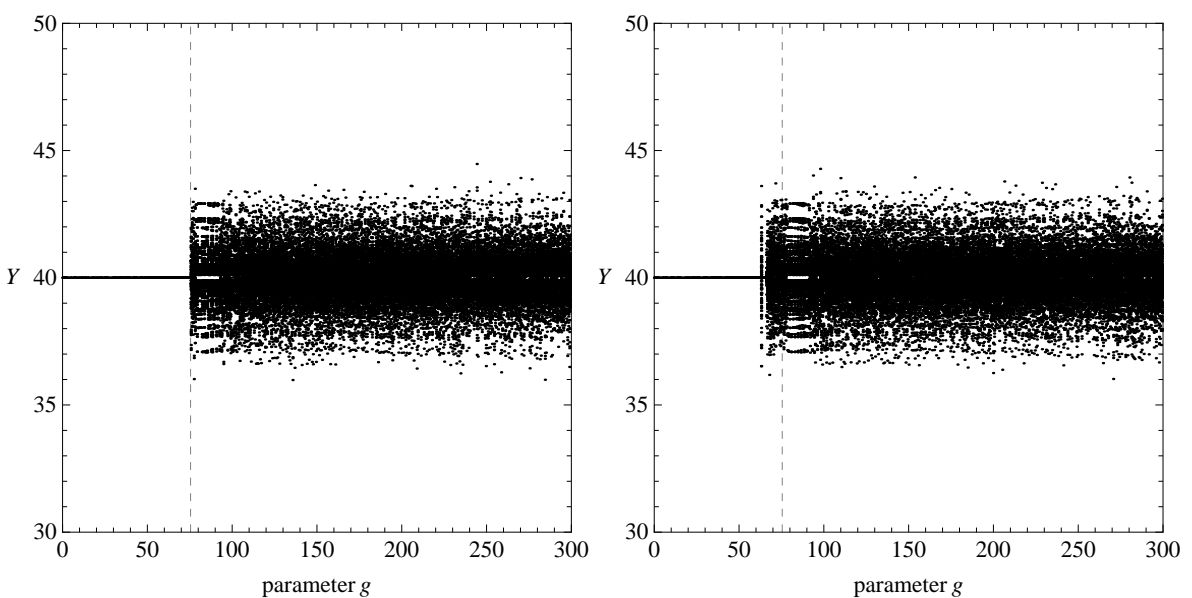

Figure 5. Bifurcation diagrams for parameter $g$ with different initial conditions. Left-hand side: $Y_{0}=40.01$. Right-hand side: $Y_{0}=40.1$. Shortly before the critical bifurcation value (depicted by the dashed line) is reached the stable fixed point coexists with a strange attractor. 


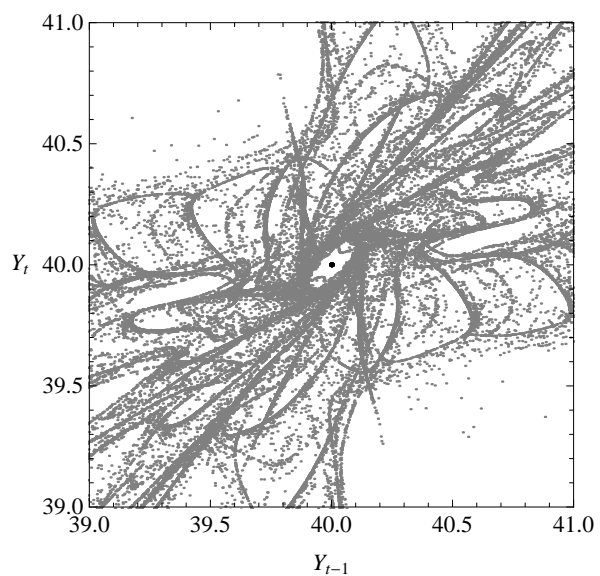

Figure 6. Shortly before the critical bifurcation value for parameter $g$ is reached, our stable fixed point (depicted in black) coexists with a strange attractor (depicted in gray). 

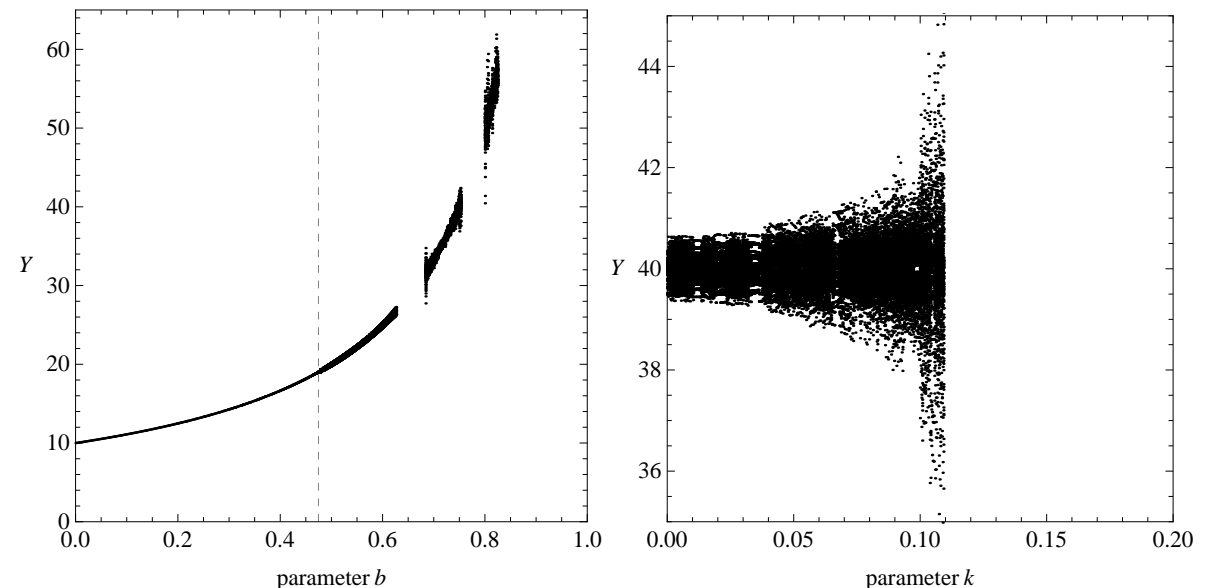

Figure \%. Bifurcation diagrams for parameters $b$ and $k$. Each parameter is increased in 500 discrete steps, as indicated on the axis. Dynamics are plotted after a transient of 5000 observations. The other parameters are identical to those in figure 1 The dashed line depicts the calculated critical bifurcation value for parameter $b$. 